\title{
Blowing Carbon Nanotubes to Carbon Nanobulbs
}

\author{
D. S. Su ${ }^{a}$, Z.P. Zhu ${ }^{\mathrm{a}, \mathrm{b}}$, Y. Lu ${ }^{\mathrm{b}}$, R. Schlögl ${ }^{\mathrm{a}}$, G. Weinberg ${ }^{\mathrm{a}}$, Z.Y. Liu ${ }^{\mathrm{b}}$ \\ a: Department of Inorganic Chemistry, Fritz Haber Institute of the MPG, Faradayweg 4-6, \\ D-14195 Berlin, Germany \\ b: State Key Laboratory of Coal Conversion, Institute of Coal Chemistry, Chinese Academy of Sciences, 030001 \\ Taiyuan, China
}

\begin{abstract}
We report the blowing of multi-walled carbon nanotubes into carbon nanobulbs. This is realized in a unique tube growth environment generated by explosive decomposition of picric acid mixed with nickel formate. The carbon spherical bulbs are characterized by large dimensions (up to $900 \mathrm{~nm}$ ), thin walls (around $10 \mathrm{~nm}$ ), and fully hollow cores. The walls are in graphitic structure of $s p^{2}$ hybridized carbons. Bulb-tube assemblies are found as intermediate derivatives of blowing. A joint action of the filled high-pressure gases and the structural defects in the carbon nanotubes is responsible to the formation of the carbon nanobulbs. Our finding may indicate the possibility to engineer the carbon nanotubes to the designed nanostructures.
\end{abstract}

\section{INTRODUCTION}

Since the discovery of fullerene molecules of carbon [1] and the report on carbon nanotube [2], carbon in various novel forms such as buckles, rings, ribbons and multiple-graphene onions has been discovered or reported [3-9]. The nanocarbons in well-shaped form are considered to be possible due to the flexibility of carbon to form pentagonal and heptagonal carbon-rings. The combination of these two basic structural units with the hexagonal carbonrings opens the world of nanocarbon with a variety of geometrical configurations. Exotic carbon nanostructures such as carbon calabashes [10-11], or carbon double helice and braids [12] are reported. Some of the non-planar carbon nanostructure can be explained by nucleation from a pentagonal atom ring or by substitution of hexagon in aromatic carbon rings by pentagons leading to curving and closed carbon structures. However, the growth mechanism for calabashes or double helices and braids still needs to be studied.

Recently, we reported on the spherical hollow carbon nanobulbs produced by an explosive decomposition of picric acid in the presence of nickel catalysts [13]. The structure of the nanobulbs is unique, characterized by large dimensions (up to $900 \mathrm{~nm}$ ), thin walls (around 10 $\mathrm{nm}$ ), and fully hollow cores. Unlike carbon spheres or carbon calabashes, the formation mechanism of carbon nanobulbs is tightly related to the thermal and mechanic properties of carbon nanotubes under special conditions. The nanobulbs are not formed directly from carbon species, rather they are derivated from carborn nanotubs by a mechanism like blowing.

\section{EXPERIMENTAL}

Picric acid and nickel formate (weight ratio of 3:1 5:1) were mixed physically and loaded into a sealed stainless steal pressure vessel $\left(10.8 \mathrm{~cm}^{3}\right)$ with a loading density of 0.25 $\mathrm{g} / \mathrm{cm}^{3}$. The decomposition reaction was induced by heating to $310{ }^{\circ} \mathrm{C}$ at a rate of $20{ }^{\circ} \mathrm{C} / \mathrm{min}$. The reaction, lasting on a microsecond scale, generated pressure of about $40 \mathrm{MPa}$ (shock wave, after which the equilibrium pressure is about $15 \mathrm{MPa}$ ) and temperature of about 930

Preprint of the Department of Inorganic Chemistry, Fritz-Haber-Institute of the MPG (for personal use only) (www.fhi-berlin.mpg.de/ac) 
${ }^{\circ} \mathrm{C}$. After the reaction, the vessel is cooled to room temperature in air and emptied of gaseous products (dominant with $\mathrm{CO}, \mathrm{CO}_{2}, \mathrm{~N}_{2}$, and $\mathrm{H}_{2} \mathrm{O}$, as revealed by gas chromatography), and the solid products are collected. For the acid treatment, $50 \mathrm{mg}$ sample was introduced into $100 \mathrm{ml}$ $6 \mathrm{M} \mathrm{HCl}$ aqueous solution and refluxed for $16 \mathrm{~h}$ with magnetic stirring. After the refluxing, the suspension was filtrated, washed with deionized water and ethanol, and dried at $110{ }^{\circ} \mathrm{C}$ overnight. SEM images were taken on a Hitachi S-4000 scanning electron microscope with a field-emission gun operated at $15-\mathrm{kV}$ accelerating voltage. TEM images were taken using a Phillips CM200 FEG electron microscope operated at $200 \mathrm{kV}$. This machine is equipped with a GATAN imaging filter GIF100 for electron energy loss spectroscopy (EELS) measurement.

\section{RESUTLS AND DISCUSSION}

Fig. 1a shows a SEM micrograph of the solid products from the decomposition of the picric acid and nickel formate mixture. Spherical structures are found along with fibre-like material. TEM investigation reveals that the fibre-like structures are multi-walled carbon nanotubes, while the spherical structures are carbon hollow nanobulbs (rather than solid carbon spheres). Most of the nanobulbs contain nickel particles formed during the decomposition of nickel formate (Fig. 1b). But few empty nanobulbs are also found in the product. The diameters of the nanobulbs vary from 100 to $900 \mathrm{~nm}$. The yield of carbon nanobulbs is about $30 \%$ of the deposits.
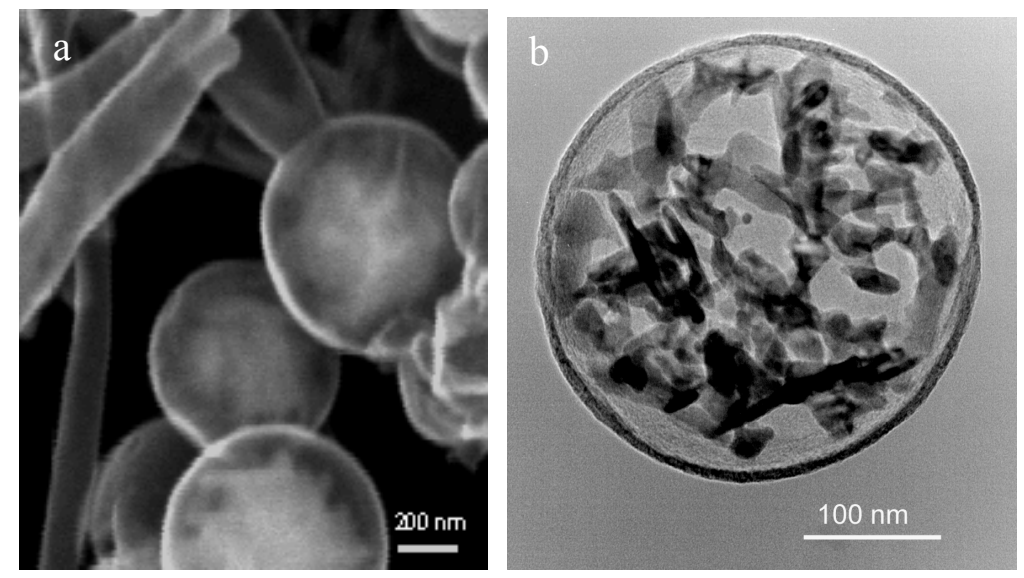

FIGURE 1. a) SEM image showing that the product contains large amount of bulbs along with carbon nanotubes. b) TEM image of a bulb containing nickel particles.

The bulbs exhibit a wall thickness in the range of 6- $25 \mathrm{~nm}$ with most of around $10 \mathrm{~nm}$. As it can be seen, the walls are uniform in thickness over the entire sphere and consist of only carbon, as revealed by EELS and EDX. Carbon $K$-edge EELS spectrum also confirms that carbon is in $s p^{2}$ hybridization state and consequently a graphitic short-range ordering. Highresolution TEM image (Fig. 2a) reveals that the walls are also well ordered in long range, constructed by an assembly of concentric graphene shells with a layer distance of $0.34 \mathrm{~nm}$. The nickel particles in the bulbs can be simply removed by treatment with $6 \mathrm{M} \mathrm{HCl}$. Fig. $2 \mathrm{~b}$ presents a typical bulb after the treatment, which clearly shows that the bulb has been completely emptied. 

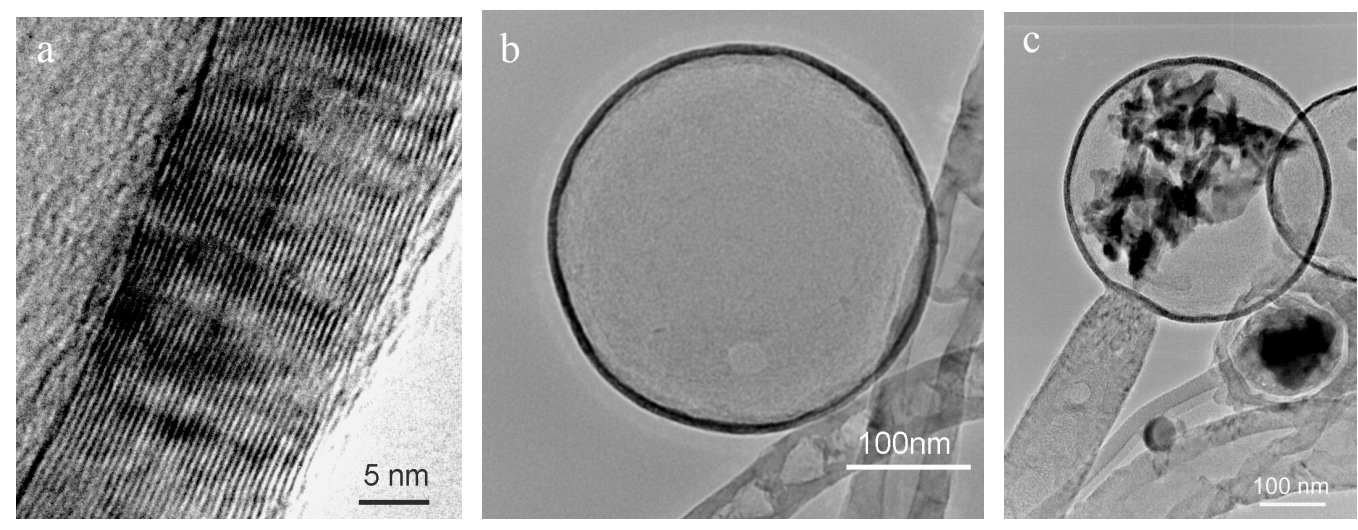

FIGURE 2. a) High-resolution TEM of a nanobulb wall; b) TEM image of an empty bulb; c) An intermediate derivative showing an bulb-tube assembly.

The obtained bulbs, characterized by large dimensions, thin walls (diameter to wallthickness ratio 100:1) and fully hollow cores are an unique carbon structure. They differ from onion-like multi-graphene shell carbon or solid carbon spheres in the hollow core. The carbon nanobulbs are also different from the graphitic hollow carbon calabashes found among the solid carbon spheres synthesized by a mixed-valent oxide-catalytic carbonization process [10], or from the carbon calabashes in a shock-produced carbon melt [11]. However, they are common in the large size and hollow-shell graphitic structure. Unlike fullerenes and solid carbon spheres, the formation and growth of nanobulbs or calabashes cannot be explained by the nucleation from a pentagonal atom ring producing an inward surface with positive curvature and therefore a spiral growth.

To understand how the bulbs are formed, we have to study the electron micrograph in Fig.1 again. Although freestanding bulbs are found, some of them connect chemically with carbon nanotubes in a flask-like structure. Bulbs at the middle of bamboo-like tubes are also found. These bulb-tube assemblies are symmetrical in their morphology along the tube axis, and the thickness of bulb walls is always thinner than the thickness of the tube wall (Fig. 2c). They can be considered as intermediate derivatives and reflect the "frozen" stages during the formation, i.e., the bulbs are formed from tubes by their volume expansion, accompanied by a decrease for the thickness of tube (or bulb) wall as the surface increases. The driving force of the expansion of the carbon nanotube could be the positive pressure difference between the inside and outside of the tube. As mentioned above, high pressure and high temperature are generated by decomposition. During the tube growth, hot and high-pressure gases fill the tube. Once the temperature outside the tubes drops after the decomposition, the temperature difference between inside and outside of the tube causes the pressure difference [13]. This induces the tube expansion and leaves behind flask-like assemblies and nanobulbs. This process is very similar to the situation in conventional glass blowing.

Beside the positive pressure difference, tubes in closed form and defects are additional conditions for the blowing. The fact that the most blowing starts on the dome of a tube could be due to the high density of defects on the cap of the tubes (for instance, pentagon rings or Stone-Walses defects). However, the blowing of tubes to the large bulbs reported here is only possible if carbon nanotubes exhibit, at least during their generation, excellent thermoplasticity and expansibility, in opposition to the usual opinion that the thermal expansion coefficient of carbon nanotubes is near zero. Our finding suggests that it is possible to engineer tubular structures at nano-scale into various shaped devices by adjusting and controlling reaction environments. 


\section{SUMMARY}

In summary, large carbon nanobulbs are produced by an explosive decomposition of picric acid at presence of nickel formate. The blowing of carbon nanotubes, a joint action of the filled high-pressure gases and the structural defects in the tubes, leads to the formation of the unique new carbon nanostructure. This finding exhibits the possibility to engineer tubular structures at nano-scale to designed forms.

\section{ACKNOWLEDGMENTS}

The work is partially supported by NSFC (59872047) and by the Deutsche Forschungsgemeinschaft (Project SCHL 332), performed in frame of ELCASS. Z. P. Zhu thanks the A.v.H. foundation for fellowship support.

\section{REFERENCES}

1. Kroto, H.W., Heath, J.R., O’Brien, S.C., Curl, R.F., Smalley, R.E., Nature 318, 162 (1985).

2. Iijima, S., Nature 354, 56 (1991).

3. Liu, J., Dai, H., Hafner, J. H., Colbert, D. T., Smalley, R. E. Nature 385, 780 (1997).

4. Sano, M., Kamino, A., Okamura, J., Shinkai, S. Science 293, 1299 (2001).

5. Martel, R., Shea, H. R., Avouris, Ph. Nature 398, 299 (1999).

6. Falvo, M. R., Clary, G. J., Taylor, R. M., Brooks, F. P., Washburn, Jr. S., Superfine, R. Nature, 389, 582 (1997).

7. Lu, J. P. Phys. Rev. Lett. 1997, 79, 1297.

8. Chopra, N. G., Benedict, L. X., Crespi, V. H., Cohen, M. L., Louie, S. G., Zettl, A. Nature 377, 135 (1995).

9. Ugarte, D. Nature 359, 707 (1992).

10 Wang. Z.L., Yin, J.S., Chem. Phys. Lett. 289, 189 (1998).

11. Rietmeijer, F.J.M.,, Schultz, P. H., Bunch, Th. E., Chem. Phys. Lett. 374, 464 (2003).

12. Lin, J., Zhang, X., Zhang, Y., Chen, X., Zhu, J., Mater. Resear. Bul. 38, 261 (2003).

13. Zhu, Z.P., Su, D.S., Lu, Y., Schlögl, R., Weinberg, G., Liu, Z.Y., Adv. Mater. 16, 443 (2004) 